
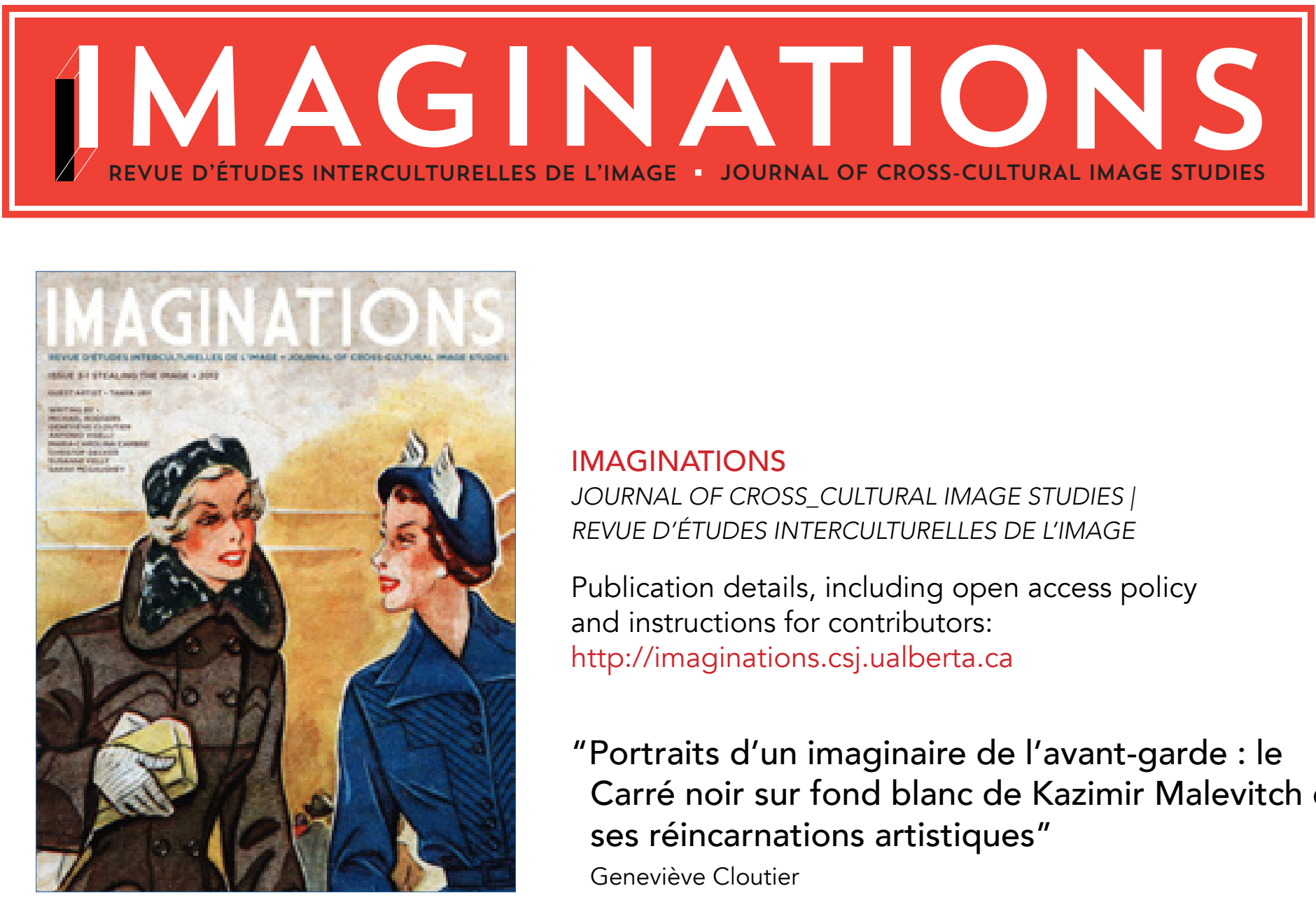

\author{
IMAGINATIONS \\ JOURNAL OF CROSS_CULTURAL IMAGE STUDIES | \\ REVUE D'ÉTUDES INTERCULTURELLES DE L'IMAGE \\ Publication details, including open access policy \\ and instructions for contributors: \\ http://imaginations.csj.ualberta.ca
}

"Portraits d'un imaginaire de l'avant-garde : le Carré noir sur fond blanc de Kazimir Malevitch et ses réincarnations artistiques"

Geneviève Cloutier

May 21, 2012

To Cite this Article:

Cloutier, Geneviève. "Portraits d'un imaginaire de l'avant-garde : le Carré noir sur fond blanc de Kazimir Malevitch et ses réincarnations artistiques" Imaginations 3:1 (2011): Web (date accessed) 30-43. DOI: 10.17742/IMAGE.stealimage.3-1.6

To Link to this article:

http://dx.doi.org/10.17742/IMAGE.stealimage.3-1.6

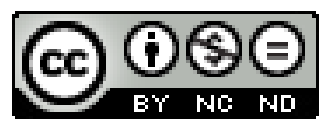

The copyright for each article belongs to the author and has been published in this journal under a Creative Commons Attribution NonCommercial NoDerivatives 3.0 license that allows others to share for non-commercial purposes the work with an acknowledgement of the work's authorship and initial publication in this journal. The content of this article represents the author's original work and any third-party content, either image or text, has been included under the Fair Dealing exception in the Canadian Copyright Act, or the author has provided the required publication permissions. 


\title{
PORTRAITS D'UN IMAGINAIRE DE L'AVANT-GARDE: LE CARRÉ NOIR SUR FOND BLANC DE KAZIMIR MALEVITCH ET SES RÉINCARNATIONS ARTISTIQUES
}

\author{
GENEVIÈVE CLOUTIER, UNIVERSITÉ DU QUÉBEC À MONTRÉAL
}

Résumé

Le Carré noir sur fond blanc de Kazimir Malevitch (1915) est l'un des tableaux les plus " récupérés » dans l'histoire récente de l'art. Avec plus ou moins de bonheur selon les cas, avec respect ou ironie, les artistes de divers horizons l'ont allègrement copié, parodié, détourné, recyclé. En Russie, l'ampleur du phénomène est telle depuis les quelque trente dernières années que, de l'art contemporain russe, on a pu dire qu'il était non pas un art après Malevitch, mais un art sur Malevitch. Dans cet article, qui prend la forme d'un parcours à travers la longue et tumultueuse histoire du Carré noir et de ses multiples "réincarnations " artistiques, je m'intéresse à ce que ces pratiques de récupération viennent nous dire sur l'attitude des artistes à l'égard du Carré noir luimême, mais aussi, et peut-être surtout, sur leur relation avec une avant-garde à l'héritage controversé dont ce tableau est devenu l'icône.

\begin{abstract}
Kazimir Malevitch's "Black Square" (1915) is one of the most recycled paintings in recent art history. More or less successfully, respectfully or ironically, artists from all around have gleefully reproduced it, ridiculed it, embezzled it and recycled it. In Russia, such has been the gravity of this phenomenon for the last thirty years, so that, it led to contemporary Russian art not being perceived as derived from Malevitch but on Malevitch. By retracing the long and tumultuous history of "Black Square" and its numerous artistic 'reincarnations,' this essay focuses on what these practices of reproduction tell us about the attitude of artists towards "Black Square" itself but also, perhaps mainly, about their relationship to an avant-garde with a controversial heritage of which this painting became the icon.
\end{abstract}


Le Carré noir sur fond blanc: réception et récupération "Un carré noir, légèrement penché, énergiquement élancé sur un fond blanc ": voici, sobrement décrit dans les mots de Victor Chklovski (Nakov, 2007:56), le contenu du tableau que Kazimir Malevitch présenta d'abord sous le titre de Quadrangle, et que l'on connaît désormais comme le Carré noir sur fond blanc. Depuis sa première apparition publique en 1915, à Petrograd, dans le cadre de la Dernière exposition futuriste 0,10, et jusqu'à aujourd'hui, près de cent ans plus tard, ce carré quelque peu irrégulier a suscité un flot presque ininterrompu de réactions et d'interprétations. Cette inflation verbale, inversement proportionnelle au dépouillement esthétique de l'œuvre, s'explique sans doute par ce dépouillement même : dès le départ, le Carré noir a été un tableau noir sur lequel les artistes et le public ont pu voir ce que leurs intérêts et leurs besoins les amenaient à projeter.

C'est Malevitch lui-même qui a initié la ronde des interprétations en disant de son œuvre qu'elle était l' « icône de notre temps » et en lui assignant par le fait même au milieu de ses autres tableaux, lors de la Dernière exposition futuriste, la place qui est traditionnellement réservée à l'icône dans l'isba russe. Les slavistes et les historiens de l'art ont amplement glosé sur ce rapprochement avec l'icône, par lequel cette œuvre à première vue iconoclaste se voyait rattachée par son auteur aux sources mêmes de la tradition picturale russe. Ce geste nous rappelle d'abord que dans la pratique de Malevitch, comme dans celle de nombreux peintres abstraits de son époque, l'art et le spirituel étaient intimement liés. Le Carré noir, comme l'icône dans la tradition orthodoxe, était pour Malevitch plus qu'un tableau; c'était une fenêtre ouverte sur une autre réalité, invisible et irreprésentable. Mais cette étiquette d' "icône de notre temps " trahit aussi les ambitions colossales du peintre qui, comme nombre de ses collègues de l'avantgarde russe, aspirait à inaugurer avec son art nouveau une ère nouvelle dans l'histoire de l'humanité. En ce sens, l'expression "icône de notre temps " évoque une époque placée sous le signe du Carré noir et du système philosophico-pictural dont Malevitch en a fait l'origine et la destination : le suprématisme.

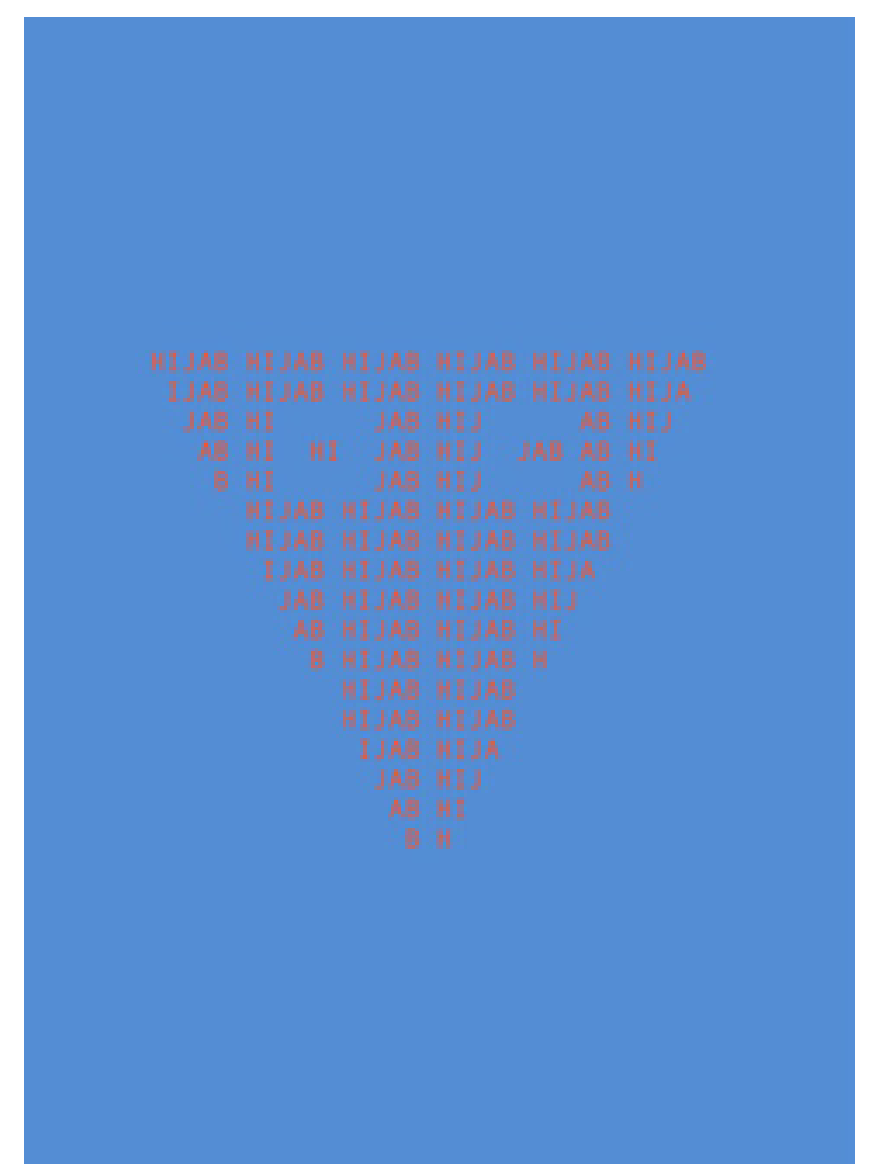

En plus d'être l'objet d'une abondante littérature critique, le Carré noir est sans aucun doute-et c'est là ce qui m'intéressera plus spécifiquement ici-l'un des tableaux les plus récupérés, sinon le tableau le plus récupéré dans l'histoire récente de l'art, et ce sur tous les modes que peut prendre la récupération. Des artistes et des designers de tous horizons l'ont allègrement copié, repris tel quel dans un geste conscient ou non, parodié et détourné (trafiqué, changé de contexte à des fins critiques ou humoristiques), cité en tant que signe chargé de sens ou recyclé, tout simplement, comme élément constituant d'autres œuvres ${ }^{1}$. Prises séparément, ces différentes œuvres d'art inspirées du Carré noir sont, il va sans dire, loin de toutes se valoir. Certaines sont désespérément simplistes ou relèvent du pillage éhonté, d'autres se 
révèlent étonnamment complexes et témoignent d'une authentique démarche créatrice. Prises toutes ensemble, pourtant, et ce quelle que soit leur valeur artistique propre, elles acquièrent un intérêt particulier dans leur manière de nous renseigner sur l'évolution, dans l'espace comme dans le temps, de l'attitude des artistes et du public à l'égard du Carré noir lui-même, bien sûr, mais aussi, et peut-être surtout, de ce qu'il représente. Car au-delà de l'exceptionnelle " ouverture » du tableau tel qu'il se présente au spectateur encore étranger aux théorisations de son auteur, au-delà, aussi, de l'ardeur de ce dernier à promouvoir son œuvre, une autre explication à la surenchère de discours autour du Carré noir réside dans le contexte historique quasi-mythique dans lequel celui-ci a été créé. En 1915, deux ans seulement avant la révolution d'Octobre, la Russie était dans un exceptionnel état d'ébullition, tant dans le domaine artistique (avec la montée des avant-gardes) que dans le domaine politique. Le Carré noir, œuvre radicale entre toutes, incarne à sa façon, et sans doute mieux que toute autre, le caractère excessif de cette époque qui a profondément marqué l'imaginaire collectif. Aussi, ce que chacun lit ou projette dans ce tableau tend à témoigner de son rapport à cette époque et à ses réalisations.

Le présent article prendra la forme d'un parcours forcément lacunaire à travers la longue et tumultueuse histoire du Carrénoir et de ses multiples « réincarnations » artistiques, principalement en Russie/Union soviétique et en (ex-)Europe de l'Est. Je m'y intéresserai surtout à la manière dont les œuvres mettant en scène le célèbre tableau de Malevitch traduisent certaines facettes d'un imaginaire collectif de l'avant-garde-imaginaire qui, dans cette partie du monde, tend d'ailleurs à incorporer un imaginaire de la Russie soviétique, comme si l'un et l'autre étaient intimement liés. Le choix d'aborder ainsi une œuvre-phare de l'avant-garde historique à travers ses multiples " récupérations ", même en employant ce terme avec neutralité, peut toutefois appeler quelques explications préliminaires. C'est que la récupération a souvent été accusée d'être cela même qui a tué l'avant-garde, dont à peu près tout le monde s'entend aujourd'hui pour dire qu'elle est morte depuis longtemps. En vertu des théories de l'avant-garde les plus communément acceptées, celle de Peter Bürger au premier chef $^{2}$, répéter les réalisations de celle-ci dans sa période dite " historique ", comme l'ont fait entre autres les représentants de la néo-avant-garde aux États-Unis dans les années soixante et comme le font les artistes et les œuvres dont il sera question ici, c'est contribuer à leur banalisation, c'est les dépouiller de leur force active, puisque ce qui relève de l'avant-garde ne peut avoir eu lieu qu'une seule fois pour toutes. La récupération s'avère ainsi néfaste et pour l'avant-garde, et pour les artistes " récupérateurs " eux-mêmes, du moins si leur ambition, en reprenant les œuvres, les idées ou les façons de faire de l'avant-garde, est de s'inscrire d'une manière ou d'une autre dans la lignée de celle-ci. Dans les années quatre-vingt et quatre-vingt-dix, toutefois, un point de vue différent sur cette question a été mis en valeur dans les travaux de Benjamin Buchloh et de Hal Foster sur la néo-avant-garde, qui ont choisi de mettre l'accent sur la part de différence qui surgit dans toute répétition ${ }^{3}$. Et si la récupération, au lieu d'être considérée comme un phénomène entièrement négatif ou, au mieux, stérile, était perçue comme un enrichissement, comme un ajout de sens par rapport à l'œuvre récupérée? Dans son essai The Return of the Real: The Avant-Garde at the End of the Century, Foster a décrit l'avant-garde, de par son aspect radical, comme un " traumatisme " qui ne peut être vraiment compris qu'après un certain délai et par l'intermédiaire de circonstances extérieures qui le ramènent à la mémoire. "[T]he avant-garde work ", écrit-il, "is never historically effective or fully significant in its initial moments. It cannot be because it is traumatic-a bole in the symbolic order of its time that is not prepared for it, that cannot receive it, at least not immediately, at least not without structural change " (29). Par rapport à l'avant-garde historique, la néoavant-garde qui, dans les années soixante, l'a empêchée de sombrer dans l'oubli et l'a remise à l'avant-plan, aurait précisément joué ce rôle d'élément déclencheur essentiel à sa pleine révélation. Ainsi, "plutôt que d'annuler l'avant-garde historique ", elle en aurait " actualis[é] le projet pour la première fois " (Foster, 1995:73). Cette manière d'envisager la réitération de l'avant-garde comme un phénomène producteur de sens et digne d'intérêt en soi est bien celle que je me propose d'adopter ici. 


\section{5 - Un sondage à la Galerie Tretiakov (en guise de} préambule)

En mars 2005, en lien avec la préparation de l'exposition "Russia! » au musée Guggenheim de New York ${ }^{4}$, un sondage fut réalisé auprès du public russe à la sortie de la galerie Tretiakov, le plus important musée d'art de Moscou. L'objet du sondage était de savoir quelle œuvre, d'une part, incarnait le mieux " l'image de la Russie » aux yeux du public et, par le fait même, méritait le plus d'être présentée à New York, et quelle œuvre, d'autre part, méritait le moins cet honneur. Les résultats de l'enquête furent éloquents : si, avec $8 \%$ des voix en sa faveur, l'icône de la Trinité d'Andreï Roublev a honorablement remporté le titre d'œuvre la plus digne de représenter la Russie, le Carré noir de Malevitch, avec un écrasant $25 \%$ de voix à son encontre, a quant à lui remporté haut la main la palme de l'œuvre jugée la moins apte à jouer ce rôle.

Dans un article consacré à ce sondage ${ }^{5}$, la revue Artkbronika présente un échantillon des commentaires faits par les répondants, qui étaient invités à expliquer leurs choix. Il en ressort, sans grande surprise, que le public russe estime reconnaître en la Trinité de Roublev (décrite entre autres comme une œuvre exceptionnellement "lumineuse ") les racines mêmes de sa propre culture. Pour ce qui est du Carré noir, en revanche, nombreux sont les répondants qui soulignent son caractère " étranger » à la culture russe et à l'image qu'ils se font de la Russie. Sa " noirceur " même est rejetée comme "non russe ", lorsqu'elle n'est pas associée à quelque chose de vaguement honteux, l'une des personnes interrogées allant jusqu'à exprimer sa crainte à l'idée que le public placé devant une telle œuvre en vienne à se représenter la Russie comme un « trou noir ». En fait, ce que ce sondage révèle, ce n'est sans doute pas tant que le Carré noir est étranger à la culture russe, mais qu'il entre en conflit avec l'image de la Russie que le public souhaite exporter. Autrement dit, l'icône correspondrait à la Russie fantasmée, tandis que le Carré noir représenterait plutôt la Russie refoulée.

On ne manquera pas de noter l'ironie du fait que le Carré noir occupe dans l'opinion publique russe le pôle

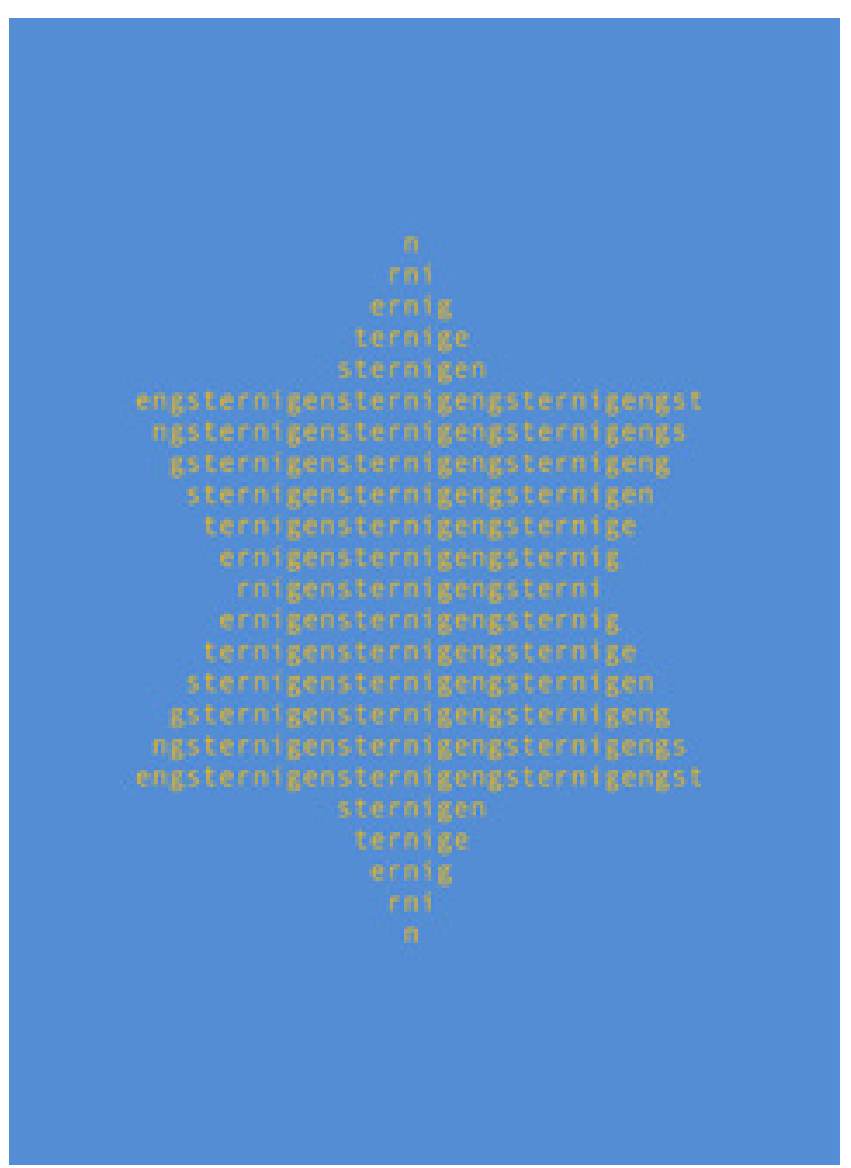

opposé par rapport à l'icône "traditionnelle » dont il prétendait justement prendre la place. Ce phénomène peut évidemment être associé à un rejet en bloc de l'art moderne ou d'avant-garde par les visiteurs de la galerie Tretiakov—un rejet dont témoigne éloquemment le fait qu'à l'exception de la Cathédrale Saint-Basile d'Aristarkh Lentulov, aucun tableau abstrait ou contemporain ne figure parmi les choix du public pour ce qui est de l'œuvre la plus digne de représenter la Russie. Il est toutefois possible de voir dans le rejet du Carré noir la manifestation d'un rejet plus généralisé de l'histoire russe du vingtième siècle. En effet, cette œuvre, de par sa prétention même à remplacer l'icône, renvoie indirectement à la profanation dont, de l'avis de plusieurs, la culture russe " authentique " aurait été victime sous le communisme. Nous verrons dans la 
suite de ce texte comment cette association du Carré au " mal russe ", étendue à l'avant-garde en général, ressort jusque dans la production artistique contemporaine.

\section{4 - La première copie}

L'interprétation du Carré noir comme signe négatif qui ressort nettement du sondage réalisé à la galerie Tretiakov remonte aussi loin que la première sortie publique de l'œuvre. À l'époque, elle fut répandue notamment par le peintre et historien de l'art Alexandre Benois, opposant notoire aux idées de l'avant-garde qui, dit-on, est à l'origine du détournement du titre du tableau de Malevitch, passé de "Quadrangle " à "Carré noir» (Nakov, 2007:63). C'est en effet dans ces mots que, dans un article critique sur l'exposition 0,10 publié dans la revue Rech', Benois désigne cette œuvre, illustration selon lui du « sermon du zéro et de la mort " de son auteur (Sharp, 42). Cette transformation du " Quadrangle " en "Carré noir " n'est pas anodine, en ce qu'elle coïncide avec la transformation du tableau comme œuvre matérielle en un objet discursif que tout le monde connaît et que plus personne n'a besoin de voir. Il est facile, en effet, de croire que tout a été dit une fois qu'on sait que Kazimir Malevitch a peint un carré noir sur fond blanc. Cependant, le Carré noir est une œuvre qui doit être mise en contexte. Présentée par Malevitch comme la clef de voûte du système suprématiste, elle doit être considérée dans la perspective de ce système ainsi que de l'ensemble de la production de l'artiste qui y est rattachée. Ainsi, Benois n'a sans doute pas tort lorsqu'il associe le Carré noir au zéro, Malevitch luimême ayant associé sa création à la notion de "zéro des formes ». La source du malentendu réside toutefois dans le sens qu'il faut donner à ce zéro. Tandis que Benois l'associe à la mort et au nihilisme, Malevitch, de son côté, en avait fait le symbole de l'économie, comprise comme l'art de la conservation de l'énergie et élevée par lui au rang de " cinquième dimension" de l'univers. La différence est de taille, entre cette compréhension du zéro comme emblème de la gestion efficace des ressources et celle du zéro comme synonyme de coupe à blanc. Il n'est d'ailleurs pas anodin de rappeler, à l'encontre de l'interprétation nihiliste du tableau, que, dans les termes de Malevitch, le Carré n'était pas seulement l'« icône de notre temps ", mais aussi l'" embryon de toutes les possibilités » et le "primogéniteur d'un monde nouveau " (Nakov, 2007:56). Encore plus éloquent à ce sujet, peut-être, est ce passage de l'article « Du cubisme et du futurisme au suprématisme-le nouveau réalisme pictural » où, après avoir associé sa découverte du Carré noir à sa propre transformation en "zéro des formes ", Malevitch affirme être arrivé " au-delà du zéro, à la création, c'est-à-dire au suprématisme » (Malevitch, 198). Dans ce texte, il apparaît clairement que le zéro, loin de se réduire à un cul-de-sac, joue dans le système malevitchien un rôle de pivot : le zéro est cela même par quoi il faut absolument passer pour aller " au-delà du zéro ", dans cet autre ordre de réalité vers lequel pointe le suprématisme ${ }^{6}$.

Andréi Nakov, dans l'imposante somme qu'il a publiée sur $\mathrm{Malevitch}^{7}$, suggère que la mésinterprétation du tableau comme simple "carré noir " arrogant et vide de sens peut aussi avoir été renforcée par les premières copies de l'œuvre, qui furent le fait de Malevitch lui-même. Cette hypothèse est intéressante dans la perspective de mon propos et mérite d'être mentionnée ici malgré son caractère quelque peu ésotérique-disons à tout le moins qu'il est difficile de la reprendre à son compte en s'appuyant uniquement sur les reproductions disponibles. Toujours est-il qu'entre 1924 et 1930-32, Malevitch s'est trouvé à devoir faire (ou à faire faire par ses élèves) trois nouvelles versions à l'identique de son Carré noir pour les besoins d'expositions diverses. Nakov attire notre attention sur le fait qu'outre leur meilleur état de conservation, ce qui frappe lorsqu'on compare les trois versions plus récentes du Carré à l'original dont elles s'inspirent, c'est leur aspect bien discipliné, tandis que dans le Carré de 1915, dont la légende veut qu'il ait été peint dans l'urgence d'une révélation ${ }^{8}$, l'expressivité prime visiblement sur le respect des principes géométriques. 


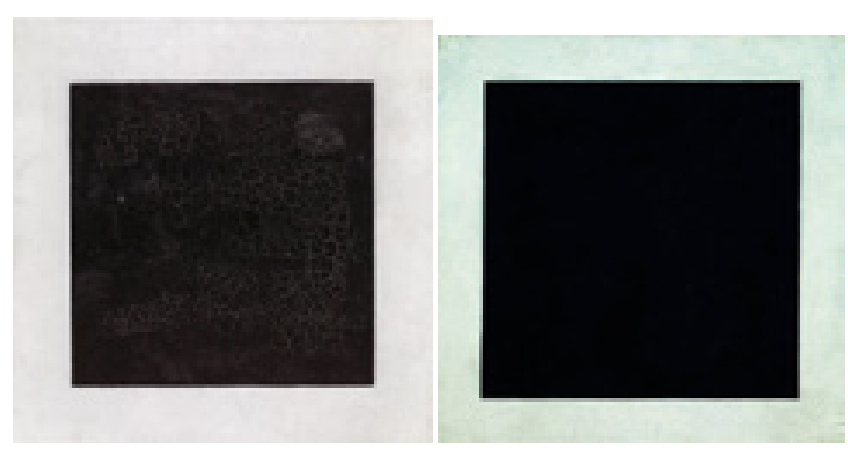

Fig. 1: Le Carré noir de 1915 et celui de 1924

De l'avis de Nakov, ces copies sont malheureuses, justement à cause de leur manque de spontanéité. Le carré y figure certes de manière plus conforme à son idéal géométrique, mais, par le fait même, il perd sa légèreté, son dynamisme, dont Chklovski avait bien su rendre compte, dans la description citée en introduction, en parlant du tableau de 1915 comme d'un carré " énergiquement élancé sur un fond blanc ». Le fait que ce dynamisme soit, a priori du moins, un aspect important du Carré noir, ressort d'ailleurs de manière évidente lorsqu'on met l'œuvre en parallèle avec les autres tableaux suprématistes de Malevitch, où les formes semblent flotter librement dans l'espace à deux dimensions qui est le leur, à l'abri de la force d'attraction terrestre. En fait, pour en revenir aux explications de Nakov, ce qui distingue les copies du Carré noir de l'original, c'est peut-être qu'elles se fondent sur un Carré déjà " mythifié ", devenu dogmatique : dans la répétition, le Carré noir se transforme en symbole infiniment reproductible d'une doctrine aux prétentions universalistes?.

\section{0 - le Carré noir mythifié et démythifié}

Le récit est connu : peu après l'arrivée au pouvoir de Staline, vers la fin des années vingt, l'avant-garde est tombée en disgrâce aux yeux du régime soviétique et ses porte-étendards ont été réduits au silence. Ainsi en fut-il du Carré noir. Pendant des décennies, son existence fut niée : il est demeuré caché dans une cave de musée, inaccessible au public. Si, à l'Ouest, on a pu

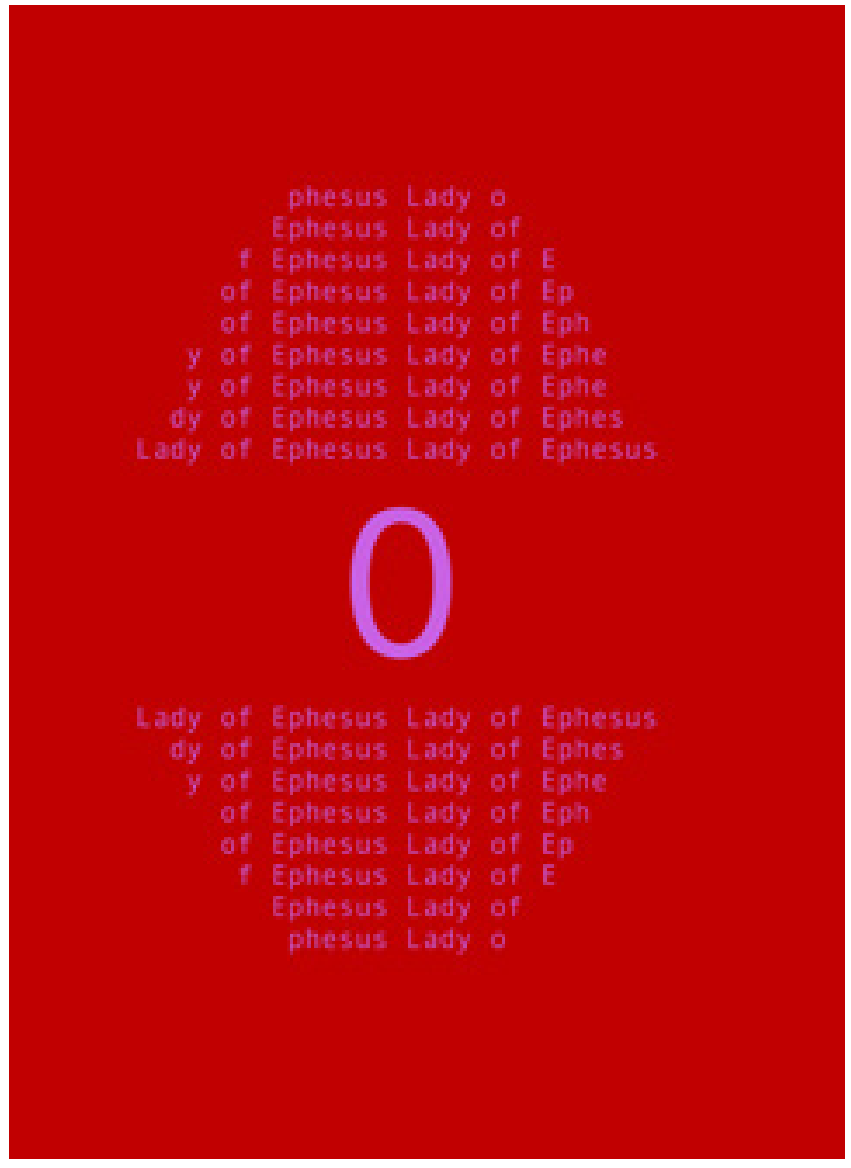

le voir ressurgir, sous une forme altérée, expurgée de tout mysticisme, dans l'esthétique minimaliste de la néoavant-garde ${ }^{10}$, en URSS, il a fallu attendre les années soixante pour que les artistes, sinon le grand public, commencent, très graduellement et très partiellement, à regagner accès à la riche production de Malevitch et de ses collègues. Cette redécouverte devait aller de pair avec l'émergence d'un véritable culte dans les milieux de l'art non conformiste, comme en témoigne entre autres la « lettre à Malevitch » publiée par le peintre Eduard Steinberg en 1981, alors que le Carré noir était enfin montré au public soviétique. Dans cette lettre, Steinberg associe le tableau de Malevitch au destin tragique de la Russie du vingtième siècle, mais il en fait son antidote plutôt que son symbole. Dans un style emphatique, traçant un portrait de la Russie soviétique comme 
d'une nation "excommunicated from Beauty, a nation numb from the broken link with the Eternal World ", il parle du "langage " de Malevitch comme d'un moyen d'exister "in the night that you call the Black Square ", et présente celui-ci comme étant cela même vers quoi " the human memory [...] will always return to [...] when mystically experiencing the tragedy of Godforsakenness " (Steinberg, 31).

Chez Steinberg, comme chez d'autres artistes associés au même milieu, cette " mythification » de l'avant-garde historique s'est traduite par une revendication explicite de son héritage, dont il croyait pouvoir reprendre le flambeau. Ses œuvres, dont on peut douter du caractère avant-gardiste mais qui se distinguent néanmoins de manière frappante de la masse de la production artistique « officielle » d'Union soviétique, se présentent ainsi comme des variations suprématistes sur le mode mineur. On y retrouve les formes géométriques chères à Malevitch, dont le carré noir, mais les couleurs sont en général moins franches : les teintes de gris, de beige, de bleu pâle, dominent là où Malevitch privilégiait les couleurs primaires. Ce choix esthétique a valu à Steinberg les railleries d'une partie de ses collègues davantage tournés vers l'art conceptuel, dont Ilia Kabakov, qui rejeta fermement cette idée selon laquelle il serait possible de reprendre le travail des avant-gardes historiques là où il a été interrompu de force, comme si rien ne s'était passé dans l'intervalle. Tandis que, chez Steinberg, l'avant-garde est perçue sous son jour héroïque, comme une production placée au-dessus du monde et non souillée par lui, Kabakov soutient que les réalisations de l'avant-garde ne peuvent plus être regardées de la même façon, dans le contexte post-stalinien, qu'à leur époque de création. Désormais, nous dit-il, le dogmatisme et l'autoritarisme des mouvements d'avant-garde font désagréablement écho aux mécanismes mêmes du régime totalitaire qui fut responsable de leur disparition.

C'est de cette manière de voir qu'est issu, dans les années soixante-dix, le mouvement du Sots Art, qui, dans une esthétique postmoderne, se caractérise par sa propension à mettre en parallèle les icônes de l'avant-garde et celles du très officiel réalisme socialiste. C'est ainsi que, dans le cadre de cette production, le Carré noir s'est vu juxtaposé notamment aux images de Staline et de l'étoile rouge (Carré noir avec liséré rouge d'Eduard Gorokhovski, 1998) et à celle de Lénine (Lénine et le Carré noir de Leonid Sokov, 2002). L'approche du Sots Art s'est rétrospectivement vu donner un fondement théorique dans les travaux de Boris Groys, dont le controversé essai Gesamtkunstwerk Stalin, publié pour la première fois en 1988, développe amplement sur cette question de la filiation entre avant-garde et totalitarisme. Reprenant l'idée communément acceptée selon laquelle, à l'origine de l'avant-garde, il y aurait une volonté d'abolir la frontière entre l'art et la vie, ou de "passer de la représentation du monde à sa transformation » (21), pour reprendre ses termes exacts, Groys y présente ce courant artistique et, au premier chef, le suprématisme malevitchien, comme une sorte de présage du stalinisme, qui, à sa différence, aurait toutefois réussi à mener à bien son projet ambitieux d'" organiser toute la vie de la société selon des formes artistiques uniques "(14). Il est toutefois important de préciser que ce lien présupposé entre l'avant-garde russe et le système qui l'a exterminée est loin d'aller de soi. Si on peut à la rigueur admettre que l'avant-garde et le totalitarisme soviétique partageaient certaines ambitions communes, si certains artistes, dans les années qui ont suivi la révolution, ont bien voulu collaborer avec le nouveau régime, séduits par l'utopie communiste, cela ne fait pas d'eux des hérauts $\mathrm{du}$ stalinisme pour autant. C'est sans mentionner, évidemment, le caractère éminemment réactionnaire de l'esthétique réaliste socialiste, qui n'a absolument rien d'une avant-garde artistique. La position consistant à amalgamer avant-garde et totalitarisme a néanmoins fait un certain nombre d'adeptes, même hors du domaine du Sots Art, comme en témoigne entre autres le titre d'un essai de l'américain d'origine bulgare Vladislav Todorov, Red Square, Black Square, où le Carré noir et la Place rouge (à laquelle l'auteur s'intéresse surtout en sa qualité de siège du mausolée de Lénine) se voient juxtaposés en tant que symboles de l'utopie soviétique.

Nombreuses sont en outre les œuvres de récupération plus ou moins récentes du Carré qui, sans faire 
directement allusion au totalitarisme soviétique, entretiennent une perception négative du suprématisme en retenant du tableau cette seule dimension vaguement autoritaire propre à la plupart des gestes et des prises de parole des mouvements d'avant-garde du début $\mathrm{du}$ vingtième siècle. Parmi celles-ci, on peut penser au tableau de Sigmar Polke intitulé «Höhere Wesen befahlen : rechte obere Ecke schwarz malen! " «Les êtres supérieurs l'ont ordonné : Peignez le coin droit supérieur en noir! ", 1969), qui fut présenté notamment lors de l'exposition Das schwarze Quadrat: Hommage an Malewitsch à Hambourg, ou encore aux multiples copies de formats variés réalisées par Allan McCollum (Plaster Surrogates, 1982-84), qui illustrent bien l'affirmation de Nakov, citée plus haut, sur le caractère dogmatique acquis par le Carré noir à force de répétition. Mais le désenchantement à l'égard de l'avant-garde et de ses promesses libératrices qui ressort de telles œuvres ne se traduit pas seulement par une mise en parallèle du Carré noir et des symboles du totalitarisme : il se traduit aussi par une tendance à associer ce tableau avec des notions ou des symboles a priori peu compatibles avec les représentations communes de l'avant-garde. La mort (avec le tableau Morte mi dano de Gerhard Merz (1988), où cette phrase est inscrite au-dessus d'un carré noir, ou encore avec l'installation plus récente Corpse of Art de Irwin (2003-2004), qui met en scène une version " épurée » d'une célèbre photographie représentant Malevitch sur son lit de mort, entouré de ses tableaux) est un thème récurrent dans les œuvres de récupération de ces dernières années, tout comme celui du commerce : la mutation du Carré noir en code-barres, par exemple, a été exécutée plus d'une fois, et c'est sans doute la version d'Alexandre Sigutin, datée de 2003, qui possède le titre le plus évocateur : Alternative au Carré noir. Comment ne pas lire de telles œuvres comme un message sur l'échec définitif, dans le monde contemporain, de l'avant-garde et de son projet ambitieux de régénérer le monde et de changer la vie?

1992 - Le Carré noir politique : le collectif Irwin et la performance Black Square on Red Square

Parmi les œuvres de récupération du Carré noir qui méritent qu'on s'y attarde un peu plus longtemps qu'aux

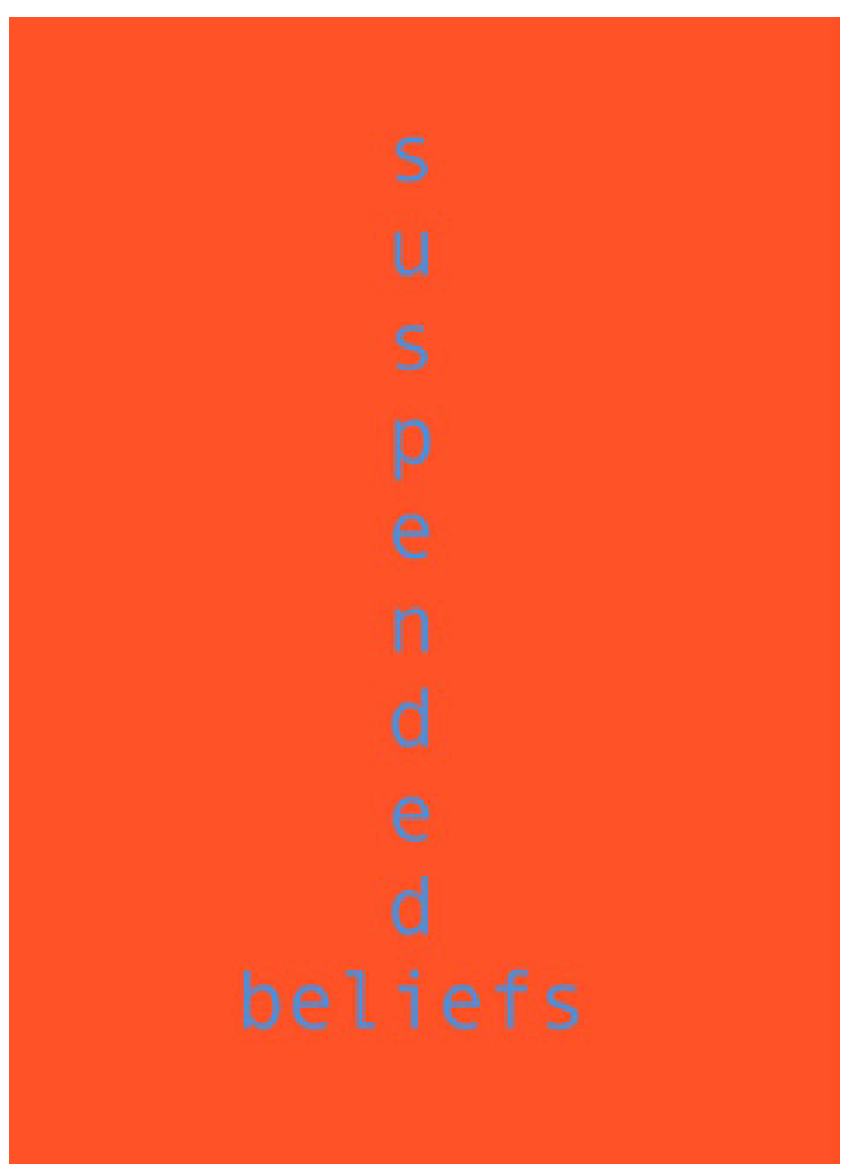

autres figure la performance Black Square on Red Square (ici encore, le titre est évidemment intraduisible si on veut préserver le parallélisme) du collectif d'artistes slovènes Irwin. L'expérience, qui eut lieu à Moscou le 6 juin 1992 et fut immortalisée sur pellicule par Michael Benson pour son film Predictions of Fire, consistait à déployer, au centre de la Place rouge, une toile noire carrée de quelque 22 mètres de côté. Nul besoin d'en savoir davantage pour comprendre la nature double, à la fois artistique et politique, d'un tel projet. Que venait donc faire un collectif d'artistes slovènes au cœur symbolique de la Russie et de l'empire soviétique, et ce en plein démantèlement de cet empire, au moment même où, partout dans le monde, et surtout dans les anciens pays communistes d'Europe, on célébrait ce démantèlement en adoptant allègrement les modèles 
de vie et d'organisation sociale venus de l'Ouest? Que venait-il faire, surtout, en y transportant le symbole par excellence de l'avant-garde russe, se l'appropriant ostensiblement?

Les réponses à ces questions sont multiples. Il faut d'abord savoir que la position du groupe Irwin dans le paysage artistique que lui-même qualifie d'est-européen (désignant par là tous les anciens pays communistes d'Europe), est une position ouvertement engagée et, sous plusieurs aspects, à contre-courant par rapport aux attitudes dominantes dans cette partie du monde depuis la chute des régimes communistes. Irwin et le collectif plus vaste dont il fait partie, Neue slowenische Kunst (NSK), ont en effet pour particularité de revendiquer ouvertement leur appartenance à cet espace esteuropéen, qu'ils considèrent comme un espace cohérent et, encore aujourd'hui, distinct par rapport au reste du monde. Depuis le début des années 2000, Irwin est derrière un vaste projet, East Art Map, qui vise à cartographier et à documenter la production artistique de cette région depuis l'après-guerre jusqu'à aujourd'hui. Le collectif est également à l'origine d'un " nouveau » courant artistique créé a posteriori, la " rétro-avantgarde ", regroupant une variété de pratiques artistiques est-européennes fondées sur une approche critique de l'avant-garde historique. Dans une intention polémique, il est même allé jusqu'à faire de ce courant l'équivalent est-européen du modernisme occidental.

La prise de position qui sous-tend ce projet controversé qu'est le East Art Map ${ }^{11}$ est un refus du discours dominant qui veut que les pratiques culturelles de l'Est aient désormais rejoint celles de l'Ouest après quelques décennies où elles auraient été écartées de la marche de l'histoire, refus qui se voit combiné à une volonté de revaloriser le parcours propre des artistes de cette partie du monde. En s'affirmant contre la tendance générale à l'homogénéisation culturelle, les membres de Irwin s'affirment aussi contre le refoulement d'une partie importante de l'histoire dont ils estiment, aussi douloureux qu'en soit le souvenir, qu'elle est constitutive de l'identité culturelle de la population est-européenne. Par ailleurs, ils soutiennent que cette histoire doit être confrontée directement si on veut éviter de voir se répéter les erreurs du passé. Replacée dans un tel contexte, la performance Black Square on Red Square, que ses auteurs n'ont accompagnée d'aucun appareil textuel, ne s'explique peut-être pas davantage d'ellemême, mais il est plus facile de cerner les enjeux qu'elle soulève, notamment en ce qui a trait au rôle de l'avantgarde historique comme élément constitutif de l'identité culturelle non seulement russe, mais est-européenne. Le fait d'aller à Moscou déployer un carré noir sur la Place rouge a sans doute, de la part d'artistes slovènes, quelque chose d'un geste de réconciliation ou, à tout le moins, de pacification, considéré dans le contexte géopolitique du début des années quatre-vingt-dix. Mais en accomplissant leur action, non seulement les membres de Irwin s'associent à l'œuvre de Malevitch, ils se posent aussi en "gardiens » du Carré. On peut donc voir cette performance comme un pied-de-nez tardif à l'endroit de la domination culturelle soviétique, comme une manière de laisser entendre que, pendant que l'URSS tentait de contrôler la culture des autres pays de l'Est, c'est là-bas que l'on préservait cet échantillon négligé de la culture russe que, désormais, Irwin s'approprie comme sien en faisant mine de le ramener cérémonieusement chez lui.

Selon Inke Arns, les membres de Irwin, en déployant leur carré noir sur la Place rouge, ont voulu confronter un puissant système idéologique avec un autre système tout aussi fort, mais dans le domaine de l'art (Arns, 9). Il y a manifestement derrière leur projet une volonté de faire ressortir ce qui est perçu comme un lien intrinsèque entre le Carré noir, la Russie et l'impérialisme soviétique et, par extension, d'attirer l'attention sur les liens qui unissent l'art et la politique. En ce sens, Black Square on Red Square s'inscrit donc dans la suite du Sots Art et de sa critique des failles, réelles ou imaginées, de l'avantgarde. Cependant, le déroulement des événements, en ce jour de juin 92, est venu donner une autre teinte à cette performance. En effet, comme le rapporte Michael Benson, alors que les participants s'attendaient à se faire interrompre par la police dès le début de leur action, ils ont pu réaliser celle-ci en toute quiétude, sous les yeux mêmes des forces de l'ordre. 
Elena Kurliandzeva has tears in her eyes. She points to a Militia member. "Go talk to him ", she says. "Finally, I can believe that things have really changed". The uniformed officer is undistinguishable from the legionaries who once cordoned off the Western embassies of Moscow. "It's a black square, it's a painting ", he explains. "I don't understand this work-but I don't see anything wrong with it ». (Arns, 196)

S'appuyant sur l'imaginaire du totalitarisme associé à la Place rouge, la performance Black Square on Red Square s'est ainsi retrouvée, contre toute attente, à célébrer à la fois la fin du totalitarisme dans une Russie en transition et la victoire de l'art sur l'idéologie.

2000 - Le Carré noir en Russie aujourd'hui : autour de quelques expositions

$\mathrm{Au}$ cours des quelques dernières années, plusieurs expositions consacrées à l'héritage du Carré noir dans les arts visuels ont eu lieu, en Russie et ailleurs, mettant en vedette des œuvres de différents pays ${ }^{12}$. Parmi cellesci, l'une, présentée à galerie moscovite Pop/Off/Art à l'été 2008, fut consacrée uniquement à son héritage dans l'art russe contemporain. Peu avant, en décembre 2006, le Art Basel de Miami Beach proposait pour sa part une exposition d'art russe intitulée "Modus R Russian Formalism Today ", qui, sans y être consacrée entièrement, accordait elle aussi une importance considérable aux nouvelles incarnations du Carré noir chez les artistes de la jeune génération. Ces expositions et la documentation qui nous en reste sont, il va sans dire, une précieuse source d'informations en ce qui a trait à la réception du Carré noir dans le monde de l'art russe. Elles ont également ceci de caractéristique qu'elles s'inscrivent-en toute conscience de la part de leurs organisateurs-dans un contexte problématique : celui de la difficile relation des Russes à leur avantgarde en général et au Carré noir en particulier. Elles traduisent toutefois une volonté explicite de revaloriser l'héritage mal-aimé de l'avant-garde, soutenue par une conviction que cet héritage est appelé à prendre une place importante dans le futur de l'art russe, voire même à jouer le rôle d'une " nouvelle " tradition dans cette

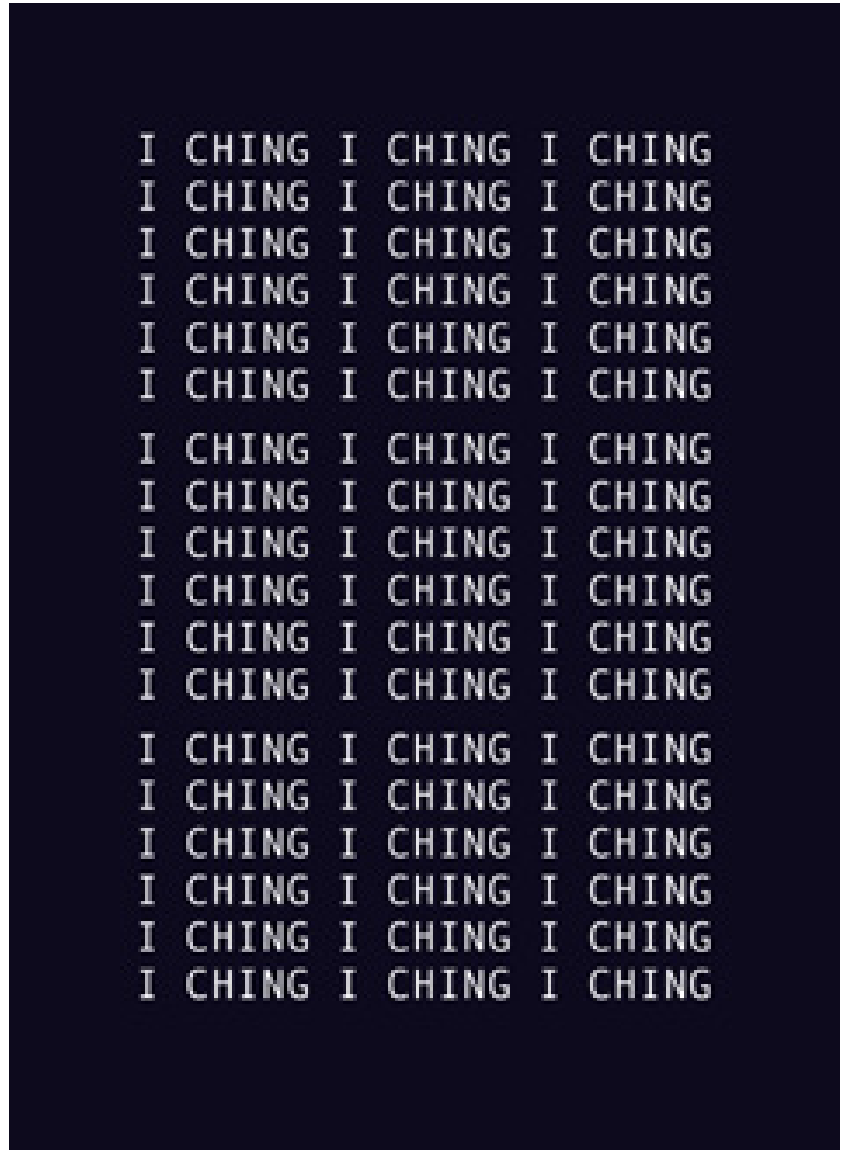

culture en quête de nouveaux symboles depuis la fin de l'époque soviétique. Sergeï Popov, le directeur de la galerie Pop/Off/Art, affirmait ainsi dans une entrevue au sujet de son « $100 \%$ Blacksquare " que, selon lui, la vitalité de l'art, de l'architecture et du design russes pour les années à venir ne pourra passer par d'autres voies que celle de la reconnaissance de l'héritage des avant-gardes historiques (" Galeristy Moskvy zanialis reklamoi "Chernogo kvadrata" Malevicha "). La commissaire de "Modus R ", Olesya Turkina, tenait en 2006 un discours semblable sur la nécessité, pour les Russes, de se réapproprier l'héritage de leur avant-garde. Mais, dans son article introductif au catalogue de l'exposition, elle soulevait aussi la question du nouveau rapport à l'avant-garde qui, selon elle, est en train de s'instaurer dans l'art actuel, par rapport à ce que l'on pouvait observer à l'époque du Sots Art. Dans ce qui 
semble être une réponse à Boris Groys, Turkina affirme que maintenant enfin, en Russie, " alors qu'apparaît une nouvelle génération d'artistes ayant grandi sans connaître l'expérience de la vie dans un état moderniste, le "politique" et le "formaliste" peuvent être séparés " ${ }^{\otimes}$. Il est vrai que, de plus en plus, les artistes qui revisitent l'héritage de l'avant-garde dans leurs œuvres semblent s'intéresser surtout à la production des artistes qui y sont associés, à certaines œuvres précises, bien plus qu'à l'avant-garde elle-même comme moment de l'histoire de l'art doté d'une lourde charge émotive, comme ce fut le cas pour les artistes des générations précédentes. Faut-il alors parler de tournant "esthétisant » dans l'approche des artistes russes contemporains à l'égard de l'avantgarde historique? L'œuvre qui occupait l'espace central de l'exposition «100\% Blacksquare », une installation d'Oleg Tatarintsev intitulée Champ (Pole), semble en tout cas aller dans ce sens. L'installation en question consistait en vingt-cinq larges bols de céramique couverts de laque noire brillante ou mate et disposés sur le plancher de manière à former un grand carré. Les propos de l'artiste au sujet de son œuvre, dans leur laconisme même, sont éloquents : "De la laque brillante et de la laque mate : cela fait contraste. Il n'y a là aucun sens caché. Vous avez dit que mon carré, c'était en fait des cercles-je n'y avais même pas pensé » ( "Metamorfozy "Chernogo kvadrata" ". Ma traduction).

L'exemple de l'œuvre Champ de Tatarintsev, sélectionnée parmi d'autres du même type, vient témoigner de ce que la volonté de faire de l'avant-garde un nouveau pilier de la culture russe ne pourra se réaliser qu'au prix d'une certaine limitation du discours à son sujet, d'une certaine aseptisation qui, manifestement, a déjà commencé à se produire. Cela n'est peut-être pas uniquement mauvais. Après toutes ces années où, sur son propre territoire, l'art de l'avant-garde russe a été subordonné à la politique, il pourrait être temps de passer à autre chose. Pour revenir aux théories de Hal Foster ainsi qu'aux propos d'Olesya Turkina cités plus haut, peut-être que l'époque contemporaine en Russie inaugure enfin celle, "post-traumatique ", où l'œuvre de l'avant-garde pourra être appréhendée dans toute sa force, libérée des interférences de la politique et de l'émotivité.

\section{Le Carré noir et l'héritage de l'avant-garde}

L'imaginaire de l'avant-garde qui se dégage des multiples œuvres de récupération du Carré noir est fait d'images fortes et parfois difficilement conciliables. Néanmoins, celles qui semblent s'imposer le plus tendent vers la formulation d'un constat d'échec de l'avant-garde comme entreprise artistique. Les idées de totalitarisme, de mort et de commerce auxquelles le Carré se voit régulièrement associé dans les œuvres de récupération incarnent sans ambiguiité l'idée de la fin de l'avant-garde, tout comme le fait, sur un autre plan toutefois, son " esthétisation " dans nombre d'œuvres contemporaines. La situation n'est pas sans être paradoxale, pourtant. C'est que malgré ce discours sur la mort de l'avant-garde dans lequel s'inscrivent les œuvres de récupération du Carré, la persistance même de ce tableau dans l'art contemporain ne manque pas d'attester de sa force d'attraction et de sa constante pertinence. Cela est particulièrement marquant dans le domaine de l'art russe. Pour montrer qu'il était encore possible de créer après le Carré, les artistes, au lieu de le reléguer aux oubliettes, se le sont approprié; ils l'ont maquillé, camouflé, profané, ridiculisé, transformé en symbole totalitaire, si bien que, pour reprendre les mots d'une historienne de l'art dans un article récent, l'art des quelques dernières décennies, en Russie, n'est peut-être pas tant un art après Malevitch qu'un art sur Malevitch (Karasik, 329). Aussi, si l'œuvre, tout comme l'image de l'avant-garde historique qu'elle incarne, a été indéniablement et durablement transformée par toutes ces manipulations, on peut néanmoins se demander où se situe le rapport de force.

Dans un article intitulé "Apropos Appropriation ", Jan Verwoert reprend cette question posée par Derrida dans Spectres de Marx: « Posséder un spectre, n'est-ce pas être possédé par lui, possédé tout court? Le capturer, n'est-ce pas être par lui captivé? " pour, à son tour, demander : "If through appropriation one seeks to (re-)possess an object, what then if that object had a history and thus a life of its own? Would the desire for possession then not inevitably be confronted by a force within that object which resists that very desire? » (Verwoert, n.p.). À notre tour, à propos du Carré noir, on peut se 
poser la question suivante, qui pourrait également être reformulée pour inclure l'avant-garde dans son entier : Est-ce l'art contemporain qui a investi le Carré noir, ou le Carré noir qui a investi l'art contemporain? ${ }^{14}$

\section{Notes}

1. Au sujet des multiples transformations subies par le Carré noir au cours de l'histoire, je renvoie à la liste dressée par Irina Karasik dans son texte introductif pour le catalogue de l'exposition The Adventures of the Black Square (Goriacheva, 29-30).

\section{Dans Theory of the Avant-Garde.}

3. À ce sujet, voir notamment l'article de Benjamin Buchloh : "The Primary Colors for the Second Time : A Paradigm Repetition of the Neo-Avant-Garde ».

4. Selon les organisateurs, cette exposition rétrospective devait présenter au public américain " the greatest masterpieces of Russian art from the 13th century to the present » («Exhibitions - Russia!»).

5. Voir : Allenova, « Ne khochu, chtoby o Rossii slozhilos vpechatlenie, kak o chernoi dyre ».

6. Rappelons à ce sujet l'intérêt qu'avait Malevitch, à l'époque où il jetait les bases du suprématisme, pour les théories de la quatrième dimension de Piotr Ouspenski.

\section{Kazimir Malewicz, le peintre absolu. 4 vols.}

8. À ce sujet, voir Nakov (Le peintre absolu 56) : «Selon les récits des élèves tardifs du peintre, la réalisation de cette peinture [...] se fit dans la souffrance d'un grandiose effort créateur. Comparable aux stéréotypes cosmogoniques des grandes expériences mystiques, ce "premier pas de la création pure dans l'art" semble avoir conduit l'artiste à un état de prostration, témoignage de l'illumination mystique à laquelle renvoyait le message de l'œuvre. La légende veut qu'épuisé par le gigantesque effort intérieur que cette création lui demandait, Malewicz ait arrêté de se nourrir et de boire (sic!) pendant plusieurs jours ».
9. Nakov écrit plus précisément, au sujet de la copie de 1924 : " Représentante quasi administrative d'un suprématisme devenu doctrinaire, cette image pour manuels scolaires franchissait ainsi le pas de la frigorification académique ». Il déplore par ailleurs le fait que ce soit «à cette façade de substitution idéologique qu'il revint jusqu'à la fin des années quatre-vingt de représenter en public l'idée malewiczéenne " (2007:75). En effet, c'est une reproduction de ce tableau, et non pas de l'original de 1915, que l'on retrouve dans les monographies qui ont révélé l'œuvre au grand public, comme The Great Experiment de Camilla Gray.

10. Sur l'héritage du Carré dans le minimalisme américain, voir entre autres Lawrence, "Back to Square One».

11. Sur le caractère controversé de East Art Map, voir par exemple le texte d'opinion de la critique d'art Nena Dimitrijevic, "Cachés derrière un rideau de fer ", publié dans la revue ArtPress. L'auteure y accuse notamment Irwin de pratiquer le "révisionnisme historique ", en trafiquant les faits pour les rendre plus conformes à leur propre version de l'histoire.

12. En plus de celles dont il sera question dans cette section, on peut mentionner Les aventures $d u$ Carré Noir, présentée en 2007 au Musée Russe de SaintPétersbourg, ainsi que Das schwarze Quadrat : Hommage an Malewitsch, présentée la même année à la Hamburger Kunsthalle.

13. Ma traduction. Au tout début du même texte, Turkina écrit également : "Modus $R$ is the trace left by modernism in contemporary Russian art. [...] The traditions of the Russian avant-garde, liberated from the ideological burden and aesthetic clichés, re-experienced today ».

14. L'image du spectre, empruntée à Derrida, a été employée par au moins deux auteurs, Wolfgang Asholt et Gérard Conio, pour parler de la persistance de l'avantgarde dans la culture contemporaine. Pour Asholt, si l'avant-garde existe encore aujourd'hui, ce n'est plus 
que sous la forme "d'un spectre, d'un fantôme qui se sert de costumes et des décors d'une autre époque, donc $\mathrm{du}$ mythe, pour pouvoir encore subsister "(Asholt, 20). Conio écrit quant à lui : " "Spectre de Marx", écrivait Derrida il y a quelques années pour constater que ce spectre pesait plus sur notre vie d'aujourd'hui que sa statue n'avait pesé sur les sociétés qui s'étaient réclamées de sa doctrine. On pourrait dire également : spectres de Maïakovski, de Khlebnikov, de Tatline, de Malévitch, de Kroutchonykh et de tous ceux qui avaient écouté l'injonction de Nietzsche : "Socrate, fais de la musique!" "(Conio, 35).

\section{Ouvrages cités}

Arns, Inke (éd.). Irwin Retroprincip. Frankfurt am Main : Revolver, 2003. Print

Asholt, Wolfgang. "L'avant-garde, le dernier mythe de l'histoire littéraire ? ". Les Mythes des avant-gardes. Éd. Véronique Léonard-Roques et Jean-Christophe Valtat. Clermont-Ferrand : Presses Universitaires Blaise Pascal, 2003. 19-32. Print.

Bessonova, Marina. "Mify russkogo avangarda v polemike pokolenii (ot Malevicha do Kabakova) » Voprosy iskusstvoznaniia 1 (1993) : 69-79. Print.

Buchloh, Benjamin. "The Primary Colors for the Second Time : A Paradigm Repetition of the Neo-AvantGarde » October 37 (Summer 1986) : 41-52. Print.

Conio, Gérard. Du gồt public : Nouveaux essais sur l'art. Lausanne : L'Âge d'homme, 2007. Print.

Dimitrijevic, Nena. "Cachés derrière un rideau de fer ". ArtPress 367 (mai 2010): 56-64.

" Exhibitions - Russia! ". [Electronic Resource]. pastexhibitions.guggenheim.org. Web. 20 Sept. 2010. <http://pastexhibitions.guggenheim.org/russia/ overview.html>

Foster, Hal. The Return of the Real : The Avant-Garde at the End of the Century. Cambridge, Mass. : MIT
Press, 1996. Print.

Foster, Hal. «Qu'en est-il du néo dans la néo-avantgarde? » Protée 23.3 (1995) : 71-79. Print.

"Galeristy Moskvy zanialis reklamoi "Chernogo kvadrata" Malevicha ". [Electronic Resource]. neusru. com. Web. 17 Nov. 2010. <http://www.newsru.com/ $\mathrm{arch} / \mathrm{cinema/23jul2008/malevich.html>}$

Goriacheva, Tatiana et. al. The Adventures of the Black Square. Saint-Pétersbourg : Palace Editions, 2007. Print.

Groys, Boris. Staline, auvre d'art totale. Trad. É. Lalliard. Nîmes : Jacqueline Chambon, 1990. Print.

Karasik, Irina. " Extending Malevich in Russian Contemporary Art ». Rethinking Malevich : Proceedings of a Conference in Celebration of the 125th Anniversary of Kazimir Malevich's Birth. Éd. Charlotte Douglas et Christina Lodder. London : Pindar Press, 2007. 328351. Print.

Malevitch, Kazimir. Écrits. Éd. A. B. Nakov. Trad. A. Robel-Chicurel. Paris : Champ Libre, 1975. Print.

" Metamorfozy "Chernogo kvadrata" v rabotakh sovremennykh khudozhnikov" [Electronic Resource]. tvkultura.ru. Web. 17 novembre 2010. <http://www. tvkultura.ru/news.html?id=245700\&cid $=46>$

Nakov, Andréi. Kazimir Malewicz, le peintre absolu. Paris : Thalia, 2007. Print.

Nakov, Andréi. Kazimir Malewicz, catalogue raisonné. Paris : A. Biro, 2002. Print.

Steinberg, Eduard. "Letter to Kazimir Malevich ». Heaven and Earth : Reflections in Paints. Éd. The State Tretyakov Gallery et. al. Saint-Pétersbourg : Palace Editions, 2004. 31. Print.

Todorov, Vladislav. Red Square, Black Square: Organon for Revolutionary Imagination. Albany : SUNY Press, 1995. Print. 
Turkina, Olesya. " Modus R : Russian Formalism Today ". Modus $R=$ Russian Formalism Today : 20 Russian Artists. Éd. Evgenija Kikodze et. al. Moskva : WAM, 2006. 18-27. " Modus R - Russia Invades Miami Art Basel ». [Electronic Resource]. scribemedia. org, Web. 18 Nov. 2010. <http://www.scribemedia. org/2007/04/28/modus-r/>

Verwoert, Jan. "Apropos Appropriation ". Art and Research 1.2 (summer 2007) : n. p. [Electronic Resource]. artandresearch.org.uk. Web. 26 Aug. 2011. $<$ http://www.artandresearch.org.uk/v1n2/verwoert. html>

\section{Bio}

Geneviève Cloutier obtained her $\mathrm{PhD}$ in Comparative Literature and Slavic Studies with a dissertation on the 'dread of history' in the Russian avant-garde. Currently, she is a post-doctoral fellow at the Figura Research Center of the Université du Québec à Montreal focusing on the re-emergence of the avant-garde in contemporary culture.

\section{Bio}

Geneviève Cloutier est titulaire d'un doctorat en études slaves et littérature comparée et auteure d'une thèse sur la " terreur de l'histoire » dans l'avant-garde russe, Elle est présentement stagiaire post-doctorale au centre de recherche Figura de l'UQAM, où elle s'intéresse aux résurgences de l'avant-garde dans la culture contemporaine. 